\title{
A NOVEL PARADIGM FOR SHORT GAMMA-RAY BURSTS WITH EXTENDED X-RAY EMISSION
}

\author{
Luciano Rezzolla ${ }^{1}$ and Pawan Kumar ${ }^{2}$ \\ ${ }^{1}$ Institute for Theoretical Physics, Frankfurt am Main, D-60438, Germany \\ ${ }^{2}$ University of Texas at Austin, Austin, TX 78712, USA \\ Received 2014 October 30; accepted 2015 January 20; published 2015 March 26
}

\begin{abstract}
The merger of a binary of neutron stars provides natural explanations for many of the features of short gamma-ray bursts (SGRBs), such as the generation of a hot torus orbiting a rapidly rotating black hole, which can then build a magnetic jet and provide the energy reservoir to launch a relativistic outflow. However, this scenario has problems explaining the recently discovered long-term and sustained X-ray emission associated with the afterglows of a subclass of SGRBs. We propose a new model that explains how an X-ray afterglow can be sustained by the product of the merger and how the X-ray emission is produced before the corresponding emission in the gammaband, though it is observed to follow it. Overall, our paradigm combines in a novel manner a number of wellestablished features of the emission in SGRBs and results from simulations. Because it involves the propagation of an ultra-relativistic outflow and its interaction with a confining medium, the paradigm also highlights a unifying phenomenology between short and long GRBs.
\end{abstract}

Key words: gamma-ray burst: general - stars: black holes - stars: magnetic field - stars: neutron stars: winds, outflows

\section{INTRODUCTION}

The merger of a binary system containing at least one neutron star (NS) represents the most attractive scenario to explain the phenomenology associated with short gamma-ray bursts (SGRBs), although many alternatives exist (see Berger 2014 for a recent review). While merging binaries of neutron stars (BNSs) were already suggested in the 1980s (Eichler et al. 1989; Narayan et al. 1992), numerical simulations (Shibata \& Uryū 2000; Anderson et al. 2008; Baiotti et al. 2008; Bernuzzi et al. 2012; Paschalidis et al. 2014) and new observations (Berger 2014) have put this scenario on firmer grounds. In particular, the simulations have shown that the merger of BNSs inevitably leads to the formation of a massive metastable object, which can either collapse promptly or survive up to a fraction of a second emitting large amounts of gravitational radiation. Furthermore, if the NSs are magnetized, the inspiral can be accompanied by a precursor electromagnetic signal (Palenzuela et al. 2013), while the merger can lead to instabilities (Siegel et al. 2013; Kiuchi et al. 2014) and to the formation of magnetically confined jet structures once a torus is formed around the black hole $(\mathrm{BH}$; Rezzolla et al. 2011; Paschalidis et al. 2014).

Despite the progress of simulations, the recent phenomenology of SGRBs presents a serious riddle for any process involving BNSs. The Swift satellite (Gehrels et al. 2004) has revealed phases of roughly constant luminosity in the X-ray afterglows of a large subclass of SGRBs. These are referred to as "X-ray plateaus" (e.g., Rowlinson et al. 2013; Gompertz et al. 2014) and last $10-10^{4} \mathrm{~s}$. The riddle is then in the timescales involved, which are too long if the X-ray emission is really an afterglow. In fact, the gamma-ray emission is normally associated to an ultra-relativistic jet launched by the $\mathrm{BH}$, produced by the collapse of the binary-merger product (BMP), in its interaction with the accreting torus. Since, the torus' mass is $\lesssim 0.1 M_{\odot}$, with accretion rates $\sim 10^{-3}-10^{-2} M_{\odot} \mathrm{m} \mathrm{s}^{-1}$ (Rezzolla et al. 2010; Hotokezaka et al. 2013), the accretion timescale is at most $\sim 1 \mathrm{~s}$. This is three or more orders of magnitude smaller than the observed timescale for the sustained X-ray emission.

An answer to this riddle is, in principle, available, but it only leads to a different one. It is possible to invoke the presence a long-lived "central engine" in terms of a "protomagnetar," that is, a uniformly rotating object formed in the merger that powers the X-ray emission through standard dipolar radiation and spindown (Zhang \& Mészáros 2001; Metzger et al. 2008, 2011; Bucciantini et al. 2012). Indeed, the BMP can either be a supramassive NS (a star with mass above the maximum mass for nonrotating configurations but below the maximum mass for uniformly rotating configurations) or a BH. The lifetime of the BMP is still very uncertain, but is likely $\lesssim 10^{4} \mathrm{~s}$ (Ravi \& Lasky 2014), which is long enough to yield a sustained X-ray emission. However, the riddle in this case is in the timing of the gamma- and $\mathrm{X}$-ray emissions. If the $\mathrm{X}$-ray emission is produced by the BMP, then it cannot follow the gamma-ray emission, which seems to require a jet and hence a BH. Indeed, none of the simulations to date indicate the generation of a collimated jet by the BMP (Price \& Rosswog 2006; Liu et al. 2008; Giacomazzo et al. 2011; Palenzuela et al. 2013; Giacomazzo et al. 2014; Kiuchi et al. 2014), which instead appears after the formation of a BH (Rezzolla et al. 2011, Dionysopoulou et al. 2015).

\section{THE BASIC PICTURE}

Before diving into the details of our "two-winds" model for those GRBs that have an X-ray afterglow, it is useful to summarize its main features. The left panel of Figure 1 presents a spacetime diagram, where shown in red is the region occupied by the BMP, which eventually collapses to produce a rapidly rotating $\mathrm{BH}$ surrounded by an accreting torus. The BMP rotates differentially for an Alfvén timescale, i.e., $\lesssim 1-10$ s and, assuming it does not collapse to a BH when differential rotation is lost, it will rotate uniformly for considerably longer, i.e., $\lesssim 10^{3}-10^{4}$ s. Shown in brown is the region occupied by the slow and baryon-rich wind, which is approximately spherical and moves at bulk speeds of 

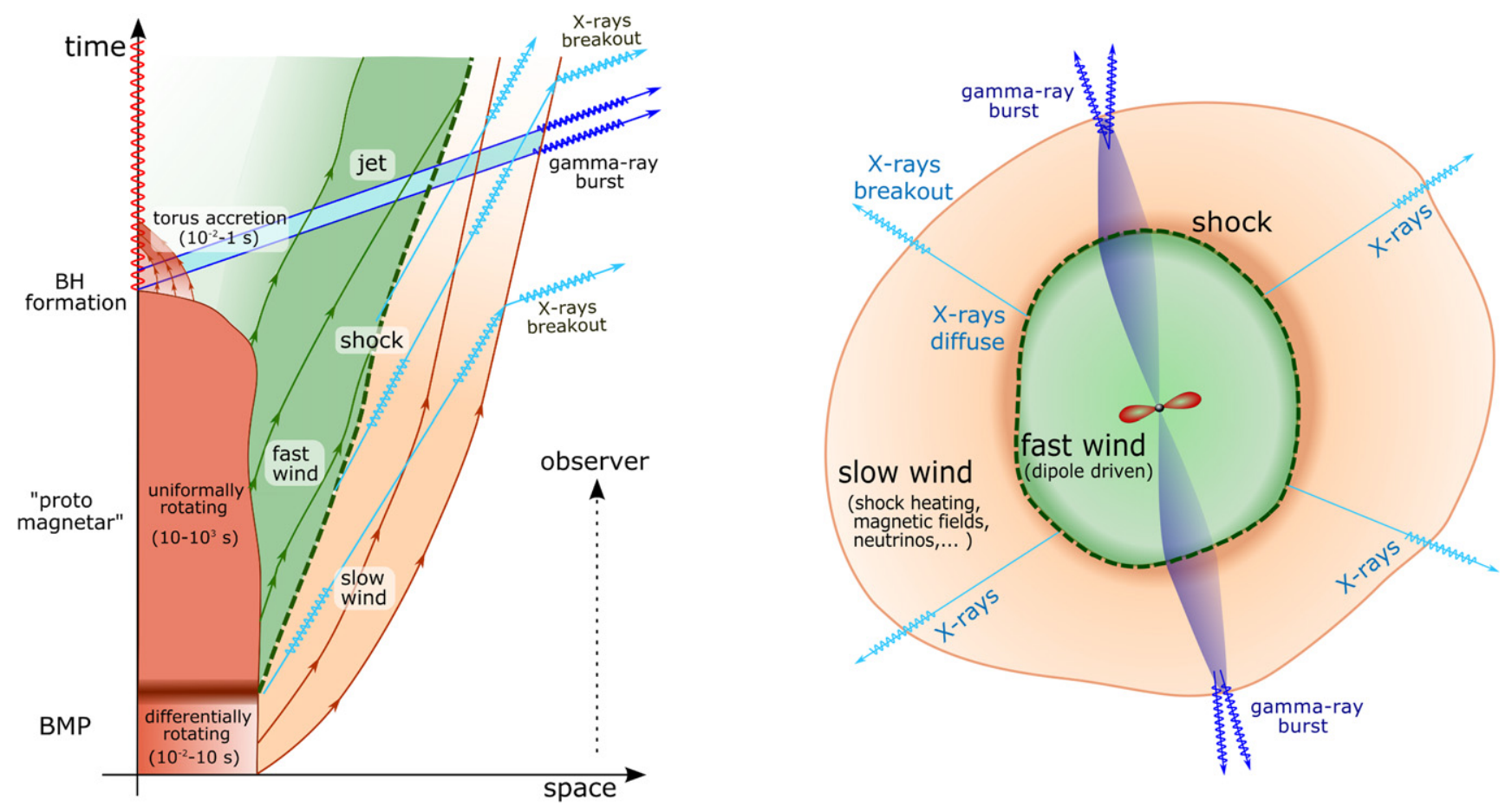

Figure 1. Left: schematic spacetime diagram showing in red the region occupied by the BMP, which eventually collapse leading to BH-torus system. Shown in brown and green are the regions occupied by the magnetically driven slow wind and by the dipole-driven fast wind. The interaction of the two winds generates a shock and the sustained X-ray emission, while a jet is produced by the BH-torus. Right: schematic snapshot after a BH-torus system has been produced and the winds have expanded.

$\sim 0.01-0.1 c$ and then progressively slows down as part of the kinetic energy is lost. This wind can be driven in a number of ways, possibly all acting at the same time: via shock heating (Hotokezaka et al. 2013), via magnetic fields and differential rotation (Kiuchi et al. 2012; Franci et al. 2013; Siegel et al. 2014), or via neutrinos (Metzger \& Fernández 2014; Perego et al. 2014). In all cases, the duration of the slow wind is $\lesssim 1-10 \mathrm{~s}$, and in the first two scenarios the wind is isotropic for realistic magnetic-field topologies (Siegel et al. 2014), and will be quenched once differential rotation is suppressed. At this point, the uniformly rotating and magnetized BMP will emit a fast and baryon-poor wind (green-shaded area) moving with bulk speeds of $\sim 0.3-0.5 c$. The BMP provides a continuous source of dipole radiation over a timescale set by the stability of the BMP, i.e., $\sim 1-10^{3} \mathrm{~s}$.

Because the slow and fast winds have different velocities, the latter catches up with the former, producing a shock that heats the matter locally and leads to an X-ray emission. However, because the matter of the slow wind is baryon rich and optically thick, the X-ray photons will not propagate freely, but rather diffuse through the slow-wind material until reaching a photospheric radius from which they reach the observer. Because the effective speed of propagation of the X-ray photons is $\sim c / \tau$, where $\tau \gg 1$ is the optical depth of the slow wind where photons are produced, and the shock front moves through the wind with a relative speed of $\sim c / 5$, X-ray diffusion can be ignored until the shock is close to the photosphere.

As the fast and slow winds interact, and the X-ray propagation takes place through the slow-wind material, the BMP will have spun down via dipolar emission to a sufficiently slow rate to collapse to a $\mathrm{BH}$ surrounded by a hot dense torus, possibly sending a radio signal (Falcke \& Rezzolla 2014; Zhang 2014). Soon after this happens, magnetic instabilities will develop in the torus, amplifying the magnetic field (Rezzolla et al. 2011; Kiuchi et al. 2014) and leading to the construction of a jet-like magnetic structure (Rezzolla et al. 2011). This magnetic funnel can then collimate the low-density material in its interior, which could be heated either by the neutrinos emitted from the torus (Ruffert \& Janka 1999) or via magnetic reconnection. In addition, the matter ejected with the slow wind can further confine the propagation of the jet (Aloy et al. 2005; Murguia-Berthier et al. 2014; Nagakura et al. 2014). As a result, an ultrarelativistic jet could be launched propagating with Lorentz factors $\Gamma \sim 100-1000$ (light-blue shaded area). The dynamics of the jet across the winds material is similar to the one envisaged for long GRBs, so that a burst of gamma-rays is assumed to be produced as the jet breaks out, with luminosities of $L \simeq 10^{50}-10^{51} \mathrm{erg} \mathrm{s}^{-1}$, over the timescale of the duration of the accreting torus, i.e., 0.01-1 s. A snapshot of the expanded winds is shown in the right panel of Figure 1.

In essence, our model solves both the X-ray timescale riddle (the emission is produced by the BMP, which can survive up to $10^{4} \mathrm{~s}$ ) and the timing riddle (the $\mathrm{X}$ - and gamma-ray emission are produced at different times and locations, and propagate at different speeds).

\section{INTERACTION OF THE SLOW AND FAST WIND AND THE RELATIVISTIC JET}

A baryon-rich slow wind is expected immediately after the merger, which lasts for a time of $t_{\mathrm{sw}} \lesssim 1-10 \mathrm{~s}$. The mass-loss rate during this time is $\dot{M}_{\mathrm{sW}} \sim 10^{-3} M_{\odot} \mathrm{s}^{-1}$ and the wind speed is $V_{\mathrm{sw}} \sim c / 10$ (Siegel et al. 2014). The slow-wind phase ends when the BMP starts to rotate as a solid body, which is also roughly when the neutrino luminosity drops off, and a global dipole magnetic field is assumed to emerge (Zhang \& 
Mészáros 2001; Metzger et al. 2008). The BMP then produces a magnetic-dipole wind with luminosity

$$
L_{d}(t) \approx\left(6 \times 10^{49} \mathrm{erg} \mathrm{s}^{-1}\right) B_{15}^{2} P_{-3}^{-4}\left(1+t / t_{\mathrm{sD}}\right)^{-2},
$$

where

$$
t_{\mathrm{sD}} \approx(500 \mathrm{~s}) B_{15}^{-2} P_{-3}^{2},
$$

is the spin-down time, $B$ is the dipole magnetic field and $P$ is BMP rotation period. After the neutrino luminosity from the BMP drops, the dipole wind is expected to have high magnetization, low baryon loading and relativistic speed. This fast wind drives a shock wave into the slow wind. The speed of the shock front moving into the slow wind, as seen in the slowwind rest-frame, can be obtained from the pressure balance as viewed in the rest-frame of the shocked wind at radius $r$

$$
\frac{L_{d}}{4 \pi r^{2} c} \approx m_{p} n_{\mathrm{sw}} V_{s}^{2} \approx \frac{\dot{M}_{\mathrm{sw}} V_{s}^{2}}{4 \pi r^{2} V_{\mathrm{sw}}},
$$

where $V_{s}$ is the shock speed as seen in the slow-wind restframe, $n_{s w}$ is the number density there, and $m_{p}$ is the unit of baryon mass. Hence,

$$
V_{s} \approx\left(\frac{L_{d} V_{\mathrm{sw}}}{\dot{M}_{\mathrm{sw}} c}\right)^{1 / 2} \approx\left(6 \times 10^{9} \mathrm{~cm} \mathrm{~s}^{-1}\right) L_{d, 50}^{1 / 2}\left(\frac{V_{\mathrm{sw}}}{c}\right)^{1 / 2} \sim V_{\mathrm{sw}} .
$$

The thermodynamic properties of the shocked slow-wind plasma can be obtained from the Rankine-Hugoniot conditions, which, for a sub-relativistic shock, yield (Rezzolla \& Zanotti 2013)

$$
\begin{gathered}
n_{\mathrm{sw}} V_{s}=n_{2} V_{2}, \\
m_{p} n_{\mathrm{sw}} V_{s}^{2}=m_{p} n_{2} V_{2}^{2}+k_{\mathrm{B}} T_{2} n_{2}+\frac{\sigma_{a} T_{2}^{4}}{3}, \\
\frac{m_{p} n_{\mathrm{sw}} V_{s}^{3}}{2}=\frac{m_{p} n_{2} V_{2}^{3}}{2}+\left[\frac{5}{2} k_{\mathrm{B}} T_{2} n_{2}+\frac{4}{3} \sigma_{a} T_{2}^{4}\right] V_{2},
\end{gathered}
$$

where $\sigma_{a}$ is the radiation constant, $k_{\mathrm{B}}$ the Boltzmann constant, and the index 2 refers to matter behind the shock. The ratio of the radiation and gas thermal pressure for the shocked slow wind is given by

$$
\sigma_{a} T_{2}^{4} /\left(3 k_{\mathrm{B}} n_{2} T_{2}\right) \sim 3 T_{2}^{3} / n_{\mathrm{sw}},
$$

When the pressure in the shocked plasma is dominated by the gas thermal pressure, we know from the standard shock-jump equations that the temperature of the shocked gas is $T_{2} \sim m_{p} V_{s}^{2} / k_{\mathrm{B}}$, which would be of the order of $10 \mathrm{MeV}$ (or $10^{11} \mathrm{~K}$ ) for the parameters of the slow and fast winds. In this case, the ratio of the radiation pressure to the gas thermal pressure is $\sim 10^{11}$ at $R=10^{10} \mathrm{~cm}$ and the ratio is $10^{13}$ at the shock break-out radius of $10^{11} \mathrm{~cm}$. This large ratio means that the pressure is completely dominated by radiation, which contradicts the assumption that the shocked plasma pressure is dominated by gas. Therefore, we must drop this assumption and consider the opposite situation in which the shocked plasma temperature is dominated by the radiation. In this case, Equations (5)-(7) yield

$$
7 V_{2}^{2}-8 V_{s} V_{2}+V_{s}^{2}=0, \quad \text { or } \quad V_{2}=V_{s} / 7
$$

Substituting this back into (5)-(6), we find

$$
n_{2}=7 n_{\mathrm{sw}}, \quad \text { and } \quad \sigma_{a} T_{2}^{4} / 3=6 m_{p} n_{\mathrm{sw}} V_{s}^{2} / 7,
$$

or

$$
T_{2} \approx\left(\frac{9 L_{d}}{14 \pi c \sigma_{a} r^{2}}\right)^{1 / 4} \sim \text { (few) } \mathrm{keV} .
$$

The X-ray luminosity from the shocked slow-wind when the shock emerges above the photosphere is obtained by solving the photon diffusion equation and is given by

$$
\begin{aligned}
L_{X} & \approx 4 \pi R_{s}^{2} \sigma_{a} T_{2}^{4}\left(\Delta r / t_{\mathrm{sw}}\right) \\
& \sim 16 \pi R_{s} \sigma_{\mathrm{SB}} T_{2}^{4}\left(R_{s} \lambda\right)^{1 / 2}\left(V_{\mathrm{sw}} / c\right)^{1 / 2},
\end{aligned}
$$

where $\sigma_{\mathrm{SB}}$ is the Stefan-Boltzmann constant, while $R_{s}=t_{\mathrm{sw}} V_{\mathrm{sw}}\left(V_{s}+V_{\mathrm{sw}}\right) / V_{s} \approx t_{\mathrm{sw}} V_{\mathrm{sw}}$ is the shock breakout radius, and

$$
\Delta r \sim\left(R_{s} \lambda c / V_{\mathrm{sw}}\right)^{1 / 2},
$$

is the radial distance travelled by the photons in $t_{s w}$ with meanfree path

$$
\lambda=\frac{4 \pi R_{s}^{2} m_{p} V_{\mathrm{sw}}}{\sigma_{\mathrm{T}} Y_{e} \dot{M}_{\mathrm{sw}}} \sim 10^{5} \mathrm{~cm},
$$

where $Y_{e} \approx 0.1$ is the electron fraction, $\sigma_{\mathrm{T}}$ the Thomsonscattering cross section, and the numerical value for $\lambda$ is for $\dot{M}_{w}=10^{-4} M_{\odot} \mathrm{s}^{-1}, V_{\mathrm{sw}}=c / 10$ and $t_{\mathrm{sw}}=30 \mathrm{~s}$. Making use of (11), we find

$$
L_{X} \sim 10^{47}\left(\mathrm{erg} \mathrm{s}^{-1}\right) \dot{M}_{w,-4}^{-1 / 2} R_{s, 12}^{1 / 2} L_{d, 50} .
$$

This luminosity is in good agreement with that observed in Xray plateaus (Rowlinson et al. 2013; Gompertz et al. 2014), and the spectrum is nonthermal because of Compton scatterings of photons below the photosphere by electrons accelerated in the shock. A part of dipole-driven energy could also be emitted in gamma-rays, as seen in some pulsars with widely ranging efficiencies $\left(10^{-3}-1\right)$ (Abdo et al. 2013). After the shock breakout, the temperature decreases due to adiabatic expansion as $r^{-2 / 3}$. The luminosity will decline as $t^{-1 / 6}$ as long as in the shocked wind $\tau>1$. At the shock, Rayleigh-Taylor instabilities may develop (Blondin et al. 2001) and particles be accelerated (Sironi \& Spitkovsky 2011).

The fast wind from the BMP lasts $t_{\mathrm{fw}} \sim 10^{3}-10^{4} \mathrm{~s}$ when the BMP loses rotational support and collapses to a $\mathrm{BH}$ and a torus. An accurate determination of the mass of this torus is difficult at the moment, as numerical simulations cannot be performed on these timescales. Previous general-relativistic simulations of the collapse of cold and uniformly rotating supramassive stars have indicated that very little mass is left outside the BH, with torus masses $\lesssim 10^{-3} M_{\odot}$ (Shibata et al. 2000; Baiotti et al. 2005, 2007), though larger masses (i.e., $10^{-2}-10^{-1} M_{\odot}$ ) can be obtained if the equation of state is sufficiently soft (Shibata 2003). The reason for this different behavior is simple to explain: with a soft equation of state the BMP will be centrally condensed and its core will collapse more rapidly than the outer parts, some of which will find themselves on stable orbits once the BH is formed. Conversely, with a stiff equation of state, the collapse will be essentially homologous, with the core and the outer parts collapsing at the same speed and leaving little material outside the BH's horizon. 
Clearly, if the mass in the torus is very small, then it will become rather difficult to find the energy reservoir needed to launch and sustain the jet that we expect in our model. However, present simulations reveal that the angular velocity distribution of the matter in the BMP has an inner core that is differentially rotating and an outer envelope that has essentially Keplerian velocities and that effectively behaves like a "disk" surrounding the BMP's core (Kastaun \& Galeazzi 2014). The amount of mass in this disk is large and can even be $30 \%$ of the total rest mass. It is then possible that the dynamics of the inner core of the BMP and that of the outer layers will be distinct. More specifically, it is not unreasonable that the inner core loses differential rotation as a result of magnetic braking on an Alfén timescale of $\lesssim 1-10 \mathrm{~s}$ and collapses to a BH much later. On the other hand, because on Keplerian orbits, the material in the outer layers could be subject to a magnetorotational instability (Velikhov 1959; Chandrasekhar 1960; Balbus \& Hawley 1991) and hence behave as a standard accretion disk onto a rapidly rotating magnetized star, in which differential rotation does not brake the rotation but transports angular momentum outwards. Once developed, magnetic turbulence will regulate accretion, which can either be on the inner core of the BMP or on the $\mathrm{BH}$ once it is formed. A good fraction of the material in the outer layers of the BMP would thus remain on quasi-Keplerian orbits on the much longer viscous accretion timescale, hence leading to the production of a massive torus around the $\mathrm{BH}$.

Following this line of arguments, which is admittedly qualitative at this stage, we assume that a relativistic jet is launched a time $t_{\mathrm{fw}}$ after the formation of BMP, and it propagates through the fast wind and the shocked slow wind. Since the fast wind and the jet are moving in the same direction at high Lorentz factors, very little work is done by the jet to open a cavity through the fast wind. However, the propagation of the jet through the slow wind, which has been at least partially shock heated by the fast wind, can require considerable expenditure of energy. This interaction also produces a hot cocoon that encapsulates and collimates the jet. Eventually, both the jet and the hot cocoon rise above the BMP slow wind surface at a radius of

$$
R_{c j} \sim t_{\mathrm{fw}} V_{s, \mathrm{sw}}\left(1+V_{s, \mathrm{sw}} / v_{h}\right),
$$

where $V_{s, \mathrm{sw}}$ is speed of the slow-wind matter at the time the cocoon punches through its surface, and $v_{h}$ is the speed of the jet head as it moves through the slow wind. Note that $V_{s, \mathrm{sw}} \sim V_{\mathrm{sw}}+V_{s} / 7$ if $R_{c j} \sim R_{s}$, but $V_{s, \mathrm{sw}} \sim V_{\mathrm{fw}}$ if the cocoon breaks out at a larger radius.

The cocoon undergoes adiabatic expansion after breaking through the BMP wind and attains mildly relativistic speed, radiating away a part of its thermal energy in the X-ray band. A far away observer will thus see a burst of gamma-rays lasting for a time of the order of the duration of the jet ( $\sim 1 \mathrm{~s}$ or less), and a long lasting phase of X-ray emission from the cocoon and from the X-ray photons produced near the shock and breaking out at the edge of the slow wind. Thermal photons from the cocoon are also inverse-Compton (IC) scattered by electrons in the jet and this could give rise to a bright $\mathrm{MeV}$ flash.

Following Ramirez-Ruiz et al. (2002), Matzner (2003), and Bromberg et al. (2011) the pressure balance between the jet and the wind in the frame comoving with the jet-head is expressed as

$$
\rho_{j} c^{2} \Gamma_{j}^{2} \Gamma_{h}^{2}\left(V_{j}-v_{h}\right)^{2} \approx\left(\rho_{2} c^{2}+4 p_{2}\right) \Gamma_{h}^{2} v_{h}^{2}+p_{2},
$$

where $\rho_{j}$ and $\rho_{2}$ are the densities of the unshocked jet and the shocked slow wind, and $\Gamma_{j}$ and $\Gamma_{h}$ are the Lorentz factors of the unshocked jet and the jet-head with respect to the slow wind. The fast wind compresses the slow wind into a thin shell of radial width $\sim R_{c j} / 7$ [Equation (10)], so that the density of the shocked slow-wind is

$$
\rho_{2} \sim \frac{t_{\mathrm{sw}} \dot{M}_{\mathrm{sw}}}{4 \pi R_{c j}^{3} / 7} \gg \frac{p_{2}}{c^{2}},
$$

Ignoring $p_{2}$ in (17), the jet-head speed relative to the shocked slow-wind medium is

$$
v_{h} \approx \frac{V_{j}}{1+\xi_{j}^{-1 / 2}},
$$

where

$$
\xi_{j} \equiv \frac{4 R_{c j} L_{j}}{7 \theta_{j}^{2} c^{3} t_{\mathrm{sw}} \dot{M}_{\mathrm{sw}}},
$$

while $L_{j}$ and $\theta_{j}$ are jet luminosity and half-opening angle, respectively. For typical parameters of $t_{\mathrm{sw}} \dot{M}_{\mathrm{sw}} \sim 10^{-3} M_{\odot}, \theta_{j} \sim 0.2, L_{j}=10^{50} \mathrm{erg} \mathrm{s}^{-1} \quad$ and $R_{c j} \sim 10^{10} \mathrm{~cm}$, we deduce that $\xi_{j} \approx 3$ and $v_{h} \sim c / 2$.

The cocoon provides collimation for the jet as long as $\xi_{j}<\theta_{j}^{-4 / 3} \quad$ (Mészáros \& Rees 2001; Bromberg et al. 2011, 2014), and, as a result, the jet angle is in general some function of $r$, which we take to be a power law.

The energy $E_{c}$ deposited by the relativistic jet into the cocoon is roughly equal to the work done by the jet on the wind medium, which is given by (Ramirez-Ruiz et al. 2002)

$$
\begin{aligned}
E_{c} \approx \int \frac{d r}{v_{h}} L_{j}\left(1-v_{h}\right) & \sim L_{j}\left(R_{c j} / 7 c\right) \\
& \sim\left(5 \times 10^{48} \mathrm{erg}\right) R_{c j, 10} L_{j, 50} .
\end{aligned}
$$

The cocoon mass and volume are, respectively,

$$
\begin{gathered}
M_{c} \approx \int d r \pi \theta_{j}^{2} r^{2} \rho_{2}(r)=\frac{7 t_{\mathrm{sw}} \dot{M}_{\mathrm{sw}}}{4} \int \frac{d r}{r} \theta_{j}^{2}, \\
\mathcal{V}_{c} \sim\left(R_{c j} / 7\right)^{3}\left(v_{\perp} / v_{h}\right)^{2},
\end{gathered}
$$

where

$$
v_{\perp} \sim\left(p_{c} / \rho_{2}\right)^{1 / 2},
$$

is the transverse expansion speed of the cocoon and $p_{c} \sim E_{c} / \mathcal{V}_{c}$ is its average thermal pressure. Combining these equations, we find

$$
v_{\perp} \sim\left[\frac{E_{c} v_{h}^{2}}{\rho_{2}}\left(\frac{7}{R_{c j}}\right)^{3}\right]^{1 / 4} .
$$

Therefore, the cocoon average temperature is given by 


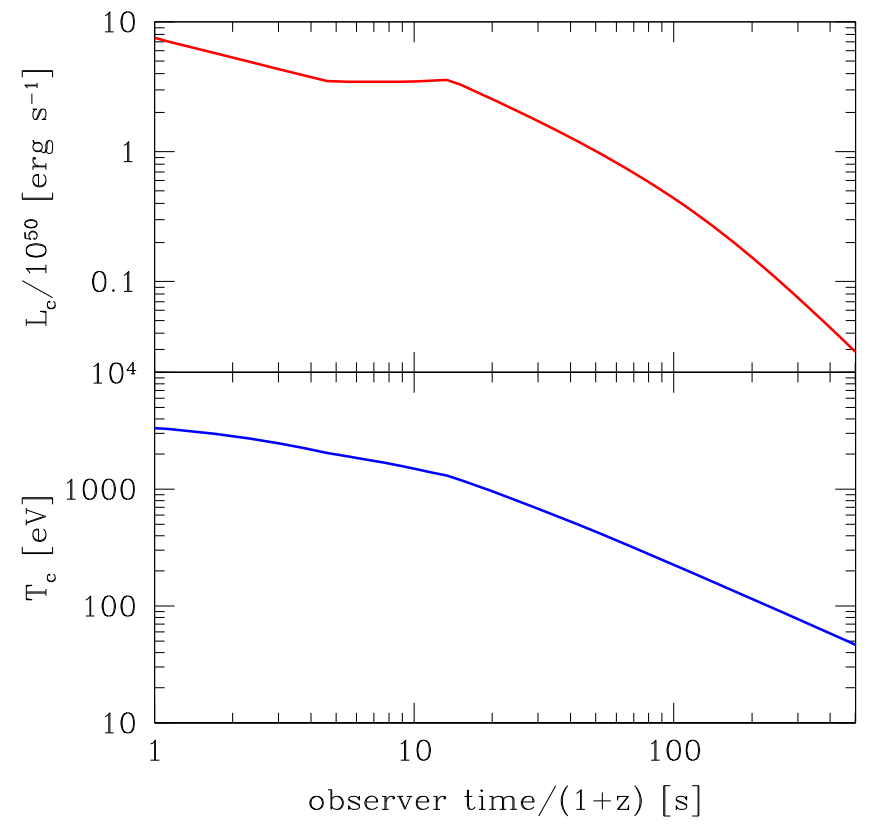

Figure 2. Upper panel shows the isotropic equivalent of luminosity of cocoon in the host-galaxy frame as a function of observer-frame time, where $z$ is the galaxy redshift (We have taken $\dot{M}_{\mathrm{sw}}=10^{-3} M_{\odot} \mathrm{s}^{-1}, V_{\mathrm{sw}}=c / 2$, and $t_{\mathrm{sw}}=10 \mathrm{~s}$ ). The lower panel shows the cocoon temperature.

$$
T_{c} \approx\left(\frac{3 p_{c}}{\sigma_{a}}\right)^{1 / 4} \sim 7 \times 10^{8} \mathrm{~K}\left[\frac{t_{\mathrm{sw}} \dot{M}_{w,-4} L_{j, 50}}{R_{c j, 10}^{5}}\right]^{1 / 8} v_{h}^{1 / 4} .
$$

Once the cocoon punches through the BMP wind, its Lorentz factor increases linearly with radius, as is the case for any radiation-dominated relativistic plasma, until it attains the terminal Lorentz factor

$$
\Gamma_{c} \approx 1+E_{c} /\left(M_{c} c^{2}\right) \sim 2-10,
$$

at a radius of $R_{\mathrm{sat}} \approx \Gamma_{c} R_{c j}$. The radiation temperature in the observer frame during this phase of acceleration does not change significantly. However, once the cocoon starts to coast at a constant speed of $v_{c}$ at $R_{\mathrm{sat}}$, the temperature decreases as $r^{-2 / 3}$. This decline steepens to $r^{-1}$ for $r \gtrsim R_{c j} \Gamma_{c}^{2}$, when the radial width of the cocoon starts to increase linearly with distance.

In the frame comoving with the cocoon, the average number density of electrons and the photon mean-free path are, respectively,

$$
n_{e, c}^{\prime}(r) \sim \frac{Y_{e} M_{c}}{m_{p} \mathcal{V}_{c}(r)}, \quad \lambda^{\prime}(r)=\frac{1}{\sigma_{T} n_{e, c}^{\prime}(r)} .
$$

The observed luminosity is controlled by photon diffusion below the photosphere, and at lab frame time $t$, this is given by

$$
L_{c}^{\text {iso }}(t) \approx 4 \pi R_{c j}^{2} \sigma_{\mathrm{SB}} T_{c}^{4}\left(R_{c j}\right)\left(\frac{\lambda^{\prime}}{c t^{\prime}+\lambda^{\prime}}\right)^{1 / 2},
$$

as long as $\lambda^{\prime}(r) \ll r / \Gamma_{c}$; here, $T_{c}\left(R_{c j}\right)$ is the cocoon temperature when it emerges above the BMP-wind surface (Equation (26)), while $t^{\prime} \sim t / \Gamma_{c}$ is the dynamical time in the cocoon rest-frame. The observed luminosity from the cocoon for $r \gtrsim \Gamma_{c}^{2} R_{c j}$ is given by

$$
L_{c}^{\text {iso }}(r) \approx 4 \pi R_{c j}^{2} \sigma_{\mathrm{SB}} T_{c}^{4}\left(R_{c j}\right) \Gamma_{c}^{4 / 3}\left(r / R_{\text {sat }}\right)^{-2}\left(\lambda^{\prime} / c t^{\prime}\right)^{1 / 2} .
$$

The cocoon luminosity and temperature are shown in Figure 2, with the luminosity being roughly constant until the radial width of the cocoon starts to increase linearly with time, which happens at $t \sim 1 \mathrm{~s}$ for our simplified cocoon model.

In reality, the decline is expected to begin later than shown in Figure 2, since the cocoon plasma is likely to continue to escape through the polar cavity at the surface for at least a few times $R_{c j} / c \sim t_{\mathrm{fw}} \sim 10^{2} \mathrm{~s}$, reducing the radial expansion of the cocoon and flattening the decay of the X-ray lightcurve. The decline steepens to $t^{-3}$ when $\tau$ of the cocoon drops below unity, which could explain the sudden drop-off at the end of the plateau in some SGRBs; the decay can be even faster if the luminosity is coming from the shock and as a result of the collapse of the BMP to a BH. Following Kumar \& Smoot (2014), we find the IC luminosity to be of the order of $L_{j}$, but that lasts only for a short duration of time of the order of $R_{c j} /\left(2 c \Gamma_{j}^{2}\right)$, since the jet is opaque at this radius and cocoon photons are only scattered when the jet first emerges above the cocoon photosphere.

\section{DISCUSSION AND SUMMARY}

The "two-winds" model proposed here pieces together events that are likely to take place when a BNS merges and provides an economical explanation for several puzzling features in the gamma-ray and X-ray data for SGRBs. The BMP is expected to be a differentially rotating highly magnetized object driving a highly baryon-loaded wind with moderate speed of $\sim 0.1 \mathrm{c}$. This phase lasts for as long as the BMP has substantial differential rotation, which might be for a few seconds. Subsequently, the baryon loading decreases and the wind becomes relativistic and is driven by magnetic-dipole radiation. This fast dipole wind pushes outward the slow baryon-rich wind and the shock resulting from their interaction heats up the plasma, generating X-ray radiation with $L_{X} \sim 10^{47} \mathrm{erg} \mathrm{s}^{-1}$ and lasting for $10^{3}-10^{4} \mathrm{~s}$ with a nearly flat light curve. This emission can explain the puzzling "plateau" seen in the X-ray light curves of SGRBs. Finally, the BMP slows down and, no longer being able to resist gravity, collapses to a $\mathrm{BH}$ leaving behind a torus of matter, which is accreted onto the $\mathrm{BH}$ in $\lesssim 1 \mathrm{~s}$. How much mass will end up in the torus is hard to predict at this stage and this represents an obvious weakness of our model. If the BMP has a stiff equation of state and collapses homologously, then it is possible that very little matter will be left outside the $\mathrm{BH}$, making the launching of a jet very problematic if possible at all. On the other hand, if the BMP has a condensed core because its equation of state is rather soft, then a non-negligible amount of matter can be used to build a torus. Clearly, new numerical simulations are needed to asses this point that may invalidate our model if it turns out that very little matter is left in the torus.

In the case in which the torus is sufficiently massive, the accreting $\mathrm{BH}$ is expected to produce a relativistic jet responsible for the observed gamma-ray emission, but the jet first has to make its way out of the baryon-loaded slow wind. As in long GRBs, the energy required for carving out a cavity through the wind is converted into thermal energy and deposited into a cocoon encapsulating the jet and contributing to its collimation. Because the fast wind has pushed the baryon- 
rich wind out to a distance of $\sim 10^{11} \mathrm{~cm}$ by the time a $\mathrm{BH}$ forms and jet is produced, the work done for clearing this cavity is much smaller than that estimated by Murguia-Berthier et al. (2014), where only a baryon-rich wind was considered. A part of the energy deposited by the jet into the cocoon is radiated away as X-rays when the jet and the cocoon rise above the wind surface, and could contribute to the extended X-ray emission. This was already recognized by Murguia-Berthier et al. (2014), although they did not calculate the emergent radiation as we have in this work. The emergent X-ray spectra in our models are nonthermal due to the interaction between photons and electrons accelerated in shocks.

The long lasting X-ray emission described in this work $\left(\sim 10^{2} \mathrm{~s}\right.$ duration $)$ arises from two different mechanisms, which are the shocked slow wind and the hot cocoon surrounding the jet. The X-ray variability times for the two mechanisms are very different. X-rays from the cocoon have a short-time variability since the cocoon has a Lorentz factor of the order of a few, and its structure is expected to be highly irregular. The timescale for X-ray luminosity from the cocoon to vary is of the order of $\delta t \sim R_{c j} /\left(2 c \Gamma_{c}^{2}\right)$, which is $\lesssim 1 \mathrm{~s}$ since $R_{c j} \sim t_{\mathrm{fw}} V_{\mathrm{sw}} \sim 10^{11} \mathrm{~cm}$. The $\mathrm{X}$-ray luminosity of the cocoon is larger than the luminosity of the shocked slow wind on a timescale of a few hundred seconds, which is the duration of observed plateau for short-GRBs, and hence the observed variability of X-rays of $\sim 1 \mathrm{~s}$ is most likely due to the cocoon emission. The variably of the X-ray luminosity from the shocked wind occurs on a longer timescale and is due to the patchiness of the material of the slow-wind. The density distribution in the slow wind is approximately spherical but not homogeneous and the shock going through it produces a hot plasma with density and temperature fluctuations. If fluctuations occur in the wind on a characteristic lengthscale of $\ell_{f}$, then the timescale for fluctuations in the emergent $\mathrm{X}$-ray lightcurve is $\sim \min \left\{\ell_{f}^{2} /(c \lambda), t_{d}\right\}$ and the dimensionless amplitude of fluctuations is $\sim\left(\ell_{f} / R_{s}\right) ; \lambda$ is photon mean-free path and $t_{d}$ is dynamical time.

While numerical simulations are still too expensive to selfconsistently reproduce this scenario, there are a number of observational features that can be used to confirm or rule out this novel paradigm. First, it is clear that in our model the launching of the jet will take place considerably after the actual merger of the two NSs, which is also when the gravitationalwave amplitude reaches its first maximum. Hence, the observation of a GRB that is seen to take place $10^{3}-10^{4} \mathrm{~s}$ after the maximum gravitational-wave emission, would be a confirmation of the validity of this scenario for SGRBs with extended X-ray emission. Second, future observations should be able to test our model by looking for IC scattered thermal cocoon photons that should show up at energies $>10 \mathrm{MeV}$ with a luminosity $\sim 10^{50} \mathrm{erg} \mathrm{s}^{-1}$ lasting for about a second. Finally, the detection of an X-ray emission anticipating the GRB would also represent a strong validation of this model; indeed the precursor signals in some SGRBs (Troja et al. 2010) seem already to suggest this possibility.

After submitting this paper, we learned about the work of Ciolfi \& Siegel (2014), who concentrate on showing that the $\mathrm{X}$-ray photon diffusion timescale is comparable or larger than the afterglow timescale. This work, on the other hand, considers the interaction between slow and fast winds, creation and propagation of a cocoon by the relativistic jet passing though the winds, and provides a detailed calculation of the resulting X-ray lightcurves. In 2013 November, L.R. and the authors had a discussion about the timing riddle, but the work has been developed entirely independently.

We are grateful to N. Bucciantini, B. Metzger, T. Piran, and B. Zhang for useful discussions and comments. Support comes from the DFG Grant SFB/Transregio 7, from "NewCompStar," COST Action MP1304, and from HIC for FAIR.

\section{REFERENCES}

Abdo, A. A., Ajello, M., Allafort, A., et al. 2013, ApJS, 208, 17

Aloy, M. A., Janka, H., \& Müller, E. 2005, A\&A, 436, 273

Anderson, M., Hirschmann, E. W., Lehner, L., et al. 2008, PhRvD, 77 024006

Baiotti, L., Giacomazzo, B., \& Rezzolla, L. 2008, PhRvD, 78, 084033

Baiotti, L., Hawke, I., Montero, P. J., et al. 2005, PhRvD, 71, 024035

Baiotti, L., Hawke, I., \& Rezzolla, L. 2007, CQGra, 24, S187

Balbus, S. A., \& Hawley, J. F. 1991, ApJ, 376, 214

Berger, E. 2014, ARA\&A, 52, 43

Bernuzzi, S., Thierfelder, M., \& Brügmann, B. 2012, PhRvD, 85, 104030

Blondin, J. M., Chevalier, R. A., \& Frierson, D. M. 2001, ApJ, 563, 806

Bromberg, O., Granot, J., Lyubarsky, Y., \& Piran, T. 2014, MNRAS, 443, 1532

Bromberg, O., Nakar, E., Piran, T., \& Sari, R. 2011, ApJ, 740, 100

Bucciantini, N., Metzger, B. D., Thompson, T. A., \& Quataert, E. 2012, MNRAS, 419, 1537

Chandrasekhar, S. 1960, PNAS, 46, 253

Ciolfi, R., \& Siegel, D. 2014, ApJL, 798, L36

Dionysopoulou, K., Alic, D., \& Rezzolla, L. 2015, arXiv:1502.02021

Eichler, D., Livio, M., Piran, T., \& Schramm, D. N. 1989, Natur, 340, 126

Falcke, H., \& Rezzolla, L. 2014, A\&A, 562, A137

Franci, L., De Pietri, R., Dionysopoulou, K., \& Rezzolla, L. 2013, PhRvD, 88, 104028

Gehrels, N., Chincarini, G., Giommi, P., et al. 2004, ApJ, 611, 1005

Giacomazzo, B., Rezzolla, L., \& Baiotti, L. 2011, PhRvD, 83, 044014

Giacomazzo, B., Zrake, J., Duffell, P., MacFadyen, A. I., \& Perna, R. 2014, arXiv: 1410.0013

Gompertz, B. P., O’Brien, P. T., \& Wynn, G. A. 2014, MNRAS, 438, 240

Hotokezaka, K., Kiuchi, K., Kyutoku, K., et al. 2013, PhRvD, 87, 024001

Kastaun, W., \& Galeazzi, F. 2014, arXiv:1411.7975

Kiuchi, K., Kyutoku, K., Sekiguchi, Y., Shibata, M., \& Wada, T. 2014 PhRvD, 90, 041502

Kiuchi, K., Kyutoku, K., \& Shibata, M. 2012, PhRvD, 86, 064008

Kumar, P., \& Smoot, G. F. 2014, MNRAS, 445, 528

Liu, Y. T., Shapiro, S. L., Etienne, Z. B., \& Taniguchi, K. 2008, PhRvD, 78, 024012

Matzner, C. D. 2003, MNRAS, 345, 575

Mészáros, P., \& Rees, M. J. 2001, ApJL, 556, L37

Metzger, B. D., \& Fernández, R. 2014, MNRAS, 441, 3444

Metzger, B. D., Giannios, D., Thompson, T. A., Bucciantini, N., \& Quataert, E. 2011, MNRAS, 413, 2031

Metzger, B. D., Quataert, E., \& Thompson, T. A. 2008, MNRAS, 385, 1455

Murguia-Berthier, A., Montes, G., Ramirez-Ruiz, E., De Colle, F., \& Lee, W. H. 2014, ApJL, 788, L8

Nagakura, H., Hotokezaka, K., Sekiguchi, Y., Shibata, M., \& Ioka, K. 2014, ApJL, 784, L28

Narayan, R., Paczynski, B., \& Piran, T. 1992, ApJL, 395, L83

Palenzuela, C., Lehner, L., Ponce, M., et al. 2013, PhRvL, 111, 061105

Paschalidis, V., Ruiz, M., \& Shapiro, S. L. 2014, arXiv:1410.7392

Perego, A., Rosswog, S., Cabezón, R. M., et al. 2014, MNRAS, 443, 3134

Price, R. H., \& Rosswog, S. 2006, Sci, 312, 719

Ramirez-Ruiz, E., Celotti, A., \& Rees, M. J. 2002, MNRAS, 337, 1349

Ravi, V., \& Lasky, P. D. 2014, MNRAS, 441, 2433

Rezzolla, L., Baiotti, L., Giacomazzo, B., Link, D., \& Font, J. A. 2010, CQGra, 27, 114105

Rezzolla, L., Giacomazzo, B., Baiotti, L., et al. 2011, ApJL, 732, L6

Rezzolla, L., \& Zanotti, O. 2013, Relativistic Hydrodynamics (Oxford: Oxford Univ. Press)

Rowlinson, A., O’Brien, P. T., Metzger, B. D., Tanvir, N. R., \& Levan, A. J. 2013, MNRAS, 430, 1061

Ruffert, M., \& Janka, H.-T. 1999, A\&A, 344, 573

Shibata, M. 2003, ApJ, 595, 992

Shibata, M., Baumgarte, T. W., \& Shapiro, S. L. 2000, PhRvD, 61, 044012 
Shibata, M., \& Uryū, K. 2000, PhRvD, 61, 064001

Siegel, D. M., Ciolfi, R., Harte, A. I., \& Rezzolla, L. 2013, PhRvD, 87, 121302

Siegel, D. M., Ciolfi, R., \& Rezzolla, L. 2014, ApJL, 785, L6

Sironi, L., \& Spitkovsky, A. 2011, ApJ, 741, 39
Troja, E., Rosswog, S., \& Gehrels, N. 2010, ApJ, 723, 1711

Velikhov, E. P. 1959, SvPh, 36, 995

Zhang, B. 2014, ApJL, 780, L21

Zhang, B., \& Mészáros, P. 2001, ApJL, 552, L35 